POS PROCEEDINGS

\title{
Polarized 3He++ Ion Source for RHIC and an EIC
}

\section{Musgrave*广and R. Milner}

Laboratory for Nuclear Science, Massachusetts Institute of Technology, Cambridge, MA, USA

E-mail: *musgrave@mit.edu

\section{G. Atoian, E. Beebe, S. Kondrashev, A. Pikin, D. Raparia, J. Ritter, and A. Zelenski}

Brookhaven National Laboratory, Upton, NY, USA

\section{J. Maxwell}

Thomas Jefferson National Accelerator Facility, Newport New, VA, USA

\begin{abstract}
The capability of accelerating a polarized ${ }^{3} \mathrm{He}$ ion beam in RHIC would demonstrate an effective polarized neutron beam for the study of new high-energy QCD studies of nucleon structure. This development would be particularly beneficial for the future plans of an Electron Ion Collider (EIC), which could use a polarized ${ }^{3} \mathrm{He}$ ion beam to probe the spin structure of the neutron. The proposed polarized ${ }^{3} \mathrm{He}$ ion source is based on the Electron Beam Ion Source (EBIS) currently in operation at Brookhaven National Laboratory (BNL). ${ }^{3} \mathrm{He}$ gas would be polarized within the $5 \mathrm{~T}$ field of the EBIS solenoid via Metastability Exchange Optical Pumping (MEOP) and then pulsed into the EBIS vacuum and drift tube system where the ${ }^{3} \mathrm{He}$ will be ionized by the $10 \mathrm{Amp}$ electron beam. The goal of the polarized ${ }^{3} \mathrm{He}$ ion source is to achieve $2.5 \times 10^{11}{ }^{3} \mathrm{He}^{++} /$pulse at $70 \%$ polarization. An upgrade of the EBIS is currently underway at BNL. The EBIS capability to produce polarized ${ }^{3} \mathrm{He}^{++}$is being developed through a collaboration between BNL and MIT.
\end{abstract}

XVII International Workshop on Polarized Sources, Targets \& Polarimetry

16-20 October 2017

Kaist, South Korea

\footnotetext{
*Speaker.

${ }^{\dagger}$ For the BNL-MIT Polarized ${ }^{3} \mathrm{He}$ Ion Source Collaboration
} 


\section{Introduction}

Discovering how the proton and neutron are composed of their constituent quarks and gluons requires experiments with a polarized electron-ion collider (EIC). The statistical precision of an EIC necessitates high intensity hadron beams with high polarization. Polarized proton beams are regularly accelerated at RHIC; however, the neutral charge of the neutron prevents its use in an accelerator. The ${ }^{3} \mathrm{He}$ nucleus has an $88.6 \%$ probability of being in its spatially symmetric S-state, where the protons form a singlet and the neutron carries the nuclear spin. This makes ${ }^{3} \mathrm{He}$ a viable surrogate for a polarized neutron beam. Polarized ${ }^{3} \mathrm{He}$ ion sources have been developed in the past $[1,2,3]$, but an EIC will require a much higher intensity and polarization than previously achieved to reach its physics goals. For this reason, development of a polarized ${ }^{3} \mathrm{He}$ ion beam has been identified as an R\&D priority for the EIC [4, 5], and the BNL-MIT polarized ${ }^{3} \mathrm{He}$ ion source collaboration has pursued this $\mathrm{R} \& \mathrm{D}[6,7,8,9,10]$.

\section{EBIS and ${ }^{3}$ He Source Overview}

The polarized ${ }^{3} \mathrm{He}$ ion source for the EIC will be based on the existing Electron Beam Ion Source (EBIS) at BNL [11, 12, 13, 14], which currently provides high charge state ions to RHIC and will remain the primary source of charged ions from D to U for an EIC based on RHIC (eRHIC). At one end of the EBIS, an electron beam is produced and then radially compressed by a $5 \mathrm{~T}$ solenoidal magnetic field before being dumped into an electron collector at the other end. The EBIS ion trap region is a series of cylindrical electrodes in the $5 \mathrm{~T}$ field. Ions are radially confined by the space charge of the electron beam and longitudinally confined by electrostatic barriers at the ends of the trap region. Ions are held in the trap until the desired charge state is reached, and then extracted by raising the potential of the trap and lowering the barrier. In the upgraded EBIS, there will be two $5 \mathrm{~T}$ solenoids. ${ }^{3} \mathrm{He}$ gas will be injected and ionized in the upstream solenoid, and ${ }^{3} \mathrm{He}$ ions will be trapped and bred to the ${ }^{3} \mathrm{He}^{++}$state in the downstream solenoid. Parameters for the EBIS production of polarized ${ }^{3} \mathrm{He}^{++}$are shown in Table 1 . Modifications that need to be made for polarized ${ }^{3} \mathrm{He}$ production include the polarization of ${ }^{3} \mathrm{He}$ at $5 \mathrm{~T}$ fields, efficient gas injection

Table 1: EBIS parameters for ${ }^{3} \mathrm{He}^{++}$

\begin{tabular}{lc}
\hline Parameter & Value \\
\hline e-beam current & $10 \mathrm{~A}$ \\
e-beam energy & $20 \mathrm{keV}$ \\
e-beam density & $\sim 575 \mathrm{~A} / \mathrm{cm}^{2}$ \\
${ }^{3} \mathrm{He}$ ion trap length & $1.5 \mathrm{~m}$ \\
Extracted beam pulse length & $\leq 20 \mu \mathrm{s}$ \\
Trap capacity (charges) & $1 \times 10^{12}$ \\
${ }^{3} \mathrm{He}^{++}$yield & $2.5-5 \times 10^{11}$ \\
${ }^{3} \mathrm{He}^{++}$polarization & $70 \%$ \\
\hline
\end{tabular}




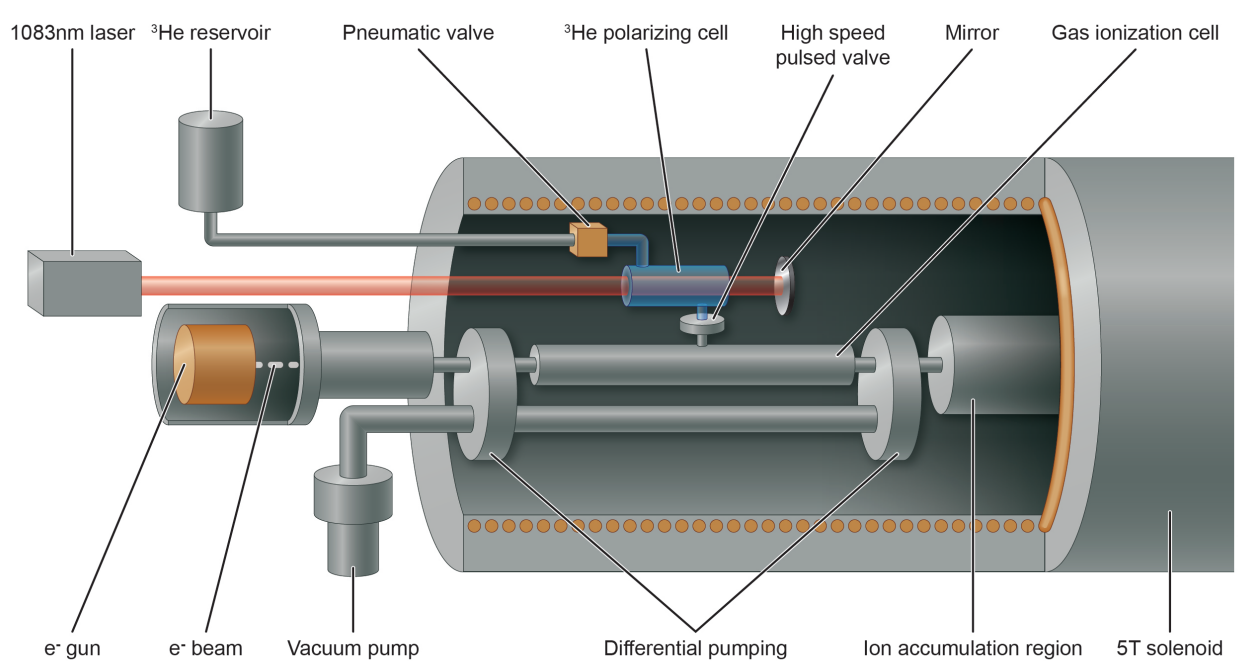

Figure 1: Conceptual illustration of the ${ }^{3} \mathrm{He}$ ion source components inside EBIS.

into the EBIS electrostatic trap, and construction of a ${ }^{3} \mathrm{He}$ spin-rotator and ${ }^{3} \mathrm{He}$ polarimeters. A conceptual illustration of primary components of the ${ }^{3} \mathrm{He}$ ion source inside the upstream end of the EBIS is shown in Figure 1.

\section{3. ${ }^{3}$ He Polarization and Purification}

The ${ }^{3} \mathrm{He}$ gas needs to be polarized before ionization to achieve the goal ${ }^{3} \mathrm{He}^{++}$polarization of $70 \%$ in RHIC. So far ${ }^{3} \mathrm{He}$ gas at 1-5 torr has been polarized in cylindrical glass cells $\left(\sim 30 \mathrm{~cm}^{3}\right)$ at 2-4 $\mathrm{T}$ magnetic fields in a spare EBIS solenoid using the technique of metastability exchange optical pumping (MEOP) [15, 16]. MEOP is performed by inducing an RF excitation of the ${ }^{3} \mathrm{He}$ gas to create a population of the $2^{3} \mathrm{~S}$ metastable state, which is optically pumped by circularly polarized $1083 \mathrm{~nm}$ light, and the nuclear spin state is simultaneously polarized by hyperfine coupling. The ${ }^{3} \mathrm{He}$ polarization is absolutely measured in the pumping cell by monitoring the transmission spectrum of a weak probe beam created by a $1083 \mathrm{~nm}$ Toptica diode laser. The maximum ${ }^{3} \mathrm{He}$ polarization is achieved in a time scale of about 10 minutes with some variation for ${ }^{3} \mathrm{He}$ pressure and purity. Preliminary results with sealed cells have measured ${ }^{3} \mathrm{He}$ polarizations near $90 \%$.

Further development with open cells attached to a gas handling system to refill and purify the ${ }^{3} \mathrm{He}$ gas have also achieved promising preliminary results with polarizations up to $73 \%$ at 3.4 torr. The open cell design uses a non-magnetic brass pneumatic valve to fill the ${ }^{3} \mathrm{He}$ cell, and to maximize the polarization, the ${ }^{3} \mathrm{He}$ gas is purified with a specialized $46 \mathrm{~K}$ cryopump to remove contaminants. A relaxation time of $30 \mathrm{~s}$ is measured in the open cell, which is limited by the metal surfaces in the pneumatic valve. Continuous $5 \mathrm{~Hz}$ operation of the EBIS will require injection of $\sim 4 \times 10^{17}{ }^{3} \mathrm{He}$ atoms per day, which corresponds to a 0.4 torr pressure drop in the $30 \mathrm{~cm}^{3}$ polarizing cell. Therefore, barring unforeseen depolarization events, a 10 minute refill and polarize period will be required once a day to maintain a polarized ${ }^{3} \mathrm{He}$ reservoir for gas injection into EBIS. 


\section{Gas Injection and Ionization}

Inside the EBIS, the ${ }^{3} \mathrm{He}$ gas will be ionized by a nominal $20 \mathrm{keV} 10 \mathrm{Amp}$ electron beam in a thin drift tube structure connected to the polarized ${ }^{3} \mathrm{He}$ reservoir by a high speed pulsed valve. A high vacuum is required outside the region of ${ }^{3} \mathrm{He}$ injection to produce a large ${ }^{3} \mathrm{He}^{++} \beta \mathrm{He}^{+}$ratio and to facilitate source recovery to generate other ion species after a 1 second switch time. The production of high charge states for heavy ions requires a vacuum in the range of $10^{-9}-10^{-10}$ torr because ambient gas will neutralize the electron beam and undergo charge exchange interactions with the desired ions, both of which inhibit the production of high charge states. Therefore, the amount of gas injected into the EBIS needs to be minimized, the region for gas ionization needs vacuum separation from the trap region for charge breeding, and the injected gas needs to be ionized or pumped out on a timescale compatible with the EBIS $5 \mathrm{~Hz}$ operation rep-rate to prevent build up of an ambient ${ }^{3} \mathrm{He}$ pressure after consecutive pulses.

A high speed pulsed valve is being developed for polarized ${ }^{3} \mathrm{He}$ injection, which opens by the Lorentz force when a current is pulsed through a conductive plate in a high magnetic field. A prototype is shown in Figure 2. The conductive plate operates as a lever arm with a pad that seals a narrow orifice to the polarized ${ }^{3} \mathrm{He}$ reservoir. Such a valve has been in successful operation in the RHIC Optically-Pumped Polarized $\mathrm{H}^{-}$Ion Source (OPPIS) source for several years [17]. The pulsed valve opens on a time scale of $\sim 500 \mu$ s and will have to inject $\sim 10^{12}{ }^{3} \mathrm{He}$ atoms to produce a uniform pressure of $10^{-6}$ torr inside a gas ionization cell with a volume of $30 \mathrm{~cm}^{3}$. A pressure limit of $10^{-6}$ torr is chosen for EBIS performance issues.

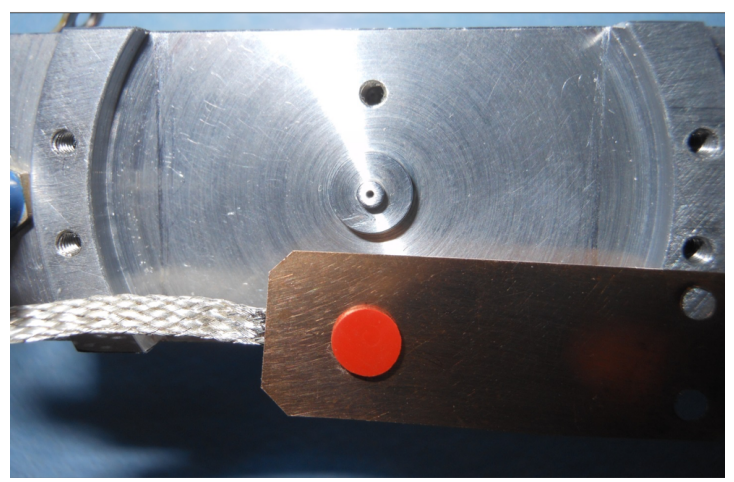

(a)

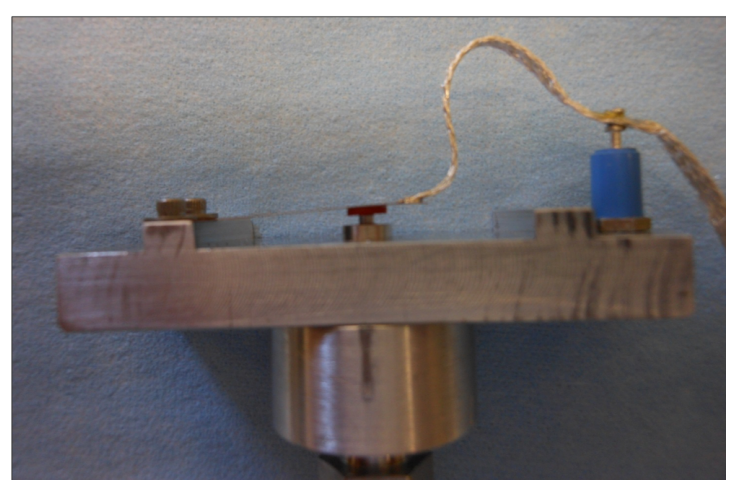

(b)

Figure 2: Prototype high speed pulsed valve for ${ }^{3} \mathrm{He}$ gas injection.

In order to minimize the amount of gas injected into the EBIS, the region of gas injection and ionization will be a narrow drift tube with constrictions on the ends to reduce gas flow conductance out of the ionization region. The feasibility of this concept was tested in the TestEBIS at BNL [18] by successfully propagating a 6 Amp electron beam through a $46 \mathrm{~cm}$ long, $1 \mathrm{~cm}$ diameter drift tube with a $3 \mathrm{~cm}$ long, $0.5 \mathrm{~cm}$ diameter constriction at one end. Another successful test performed with the TestEBIS was the ionization and extraction of ${ }^{4} \mathrm{He}$ ions after creating a residual ${ }^{4} \mathrm{He}$ pressure in the TestEBIS vacuum system. A necessary future test will be the ionization and extraction of ${ }^{4} \mathrm{He}$ $\&{ }^{3} \mathrm{He}$ injected with the high speed pulsed valve. Simulations are being conducted to prepare for gas injection tests and to optimize the gas injection and ionization system. 


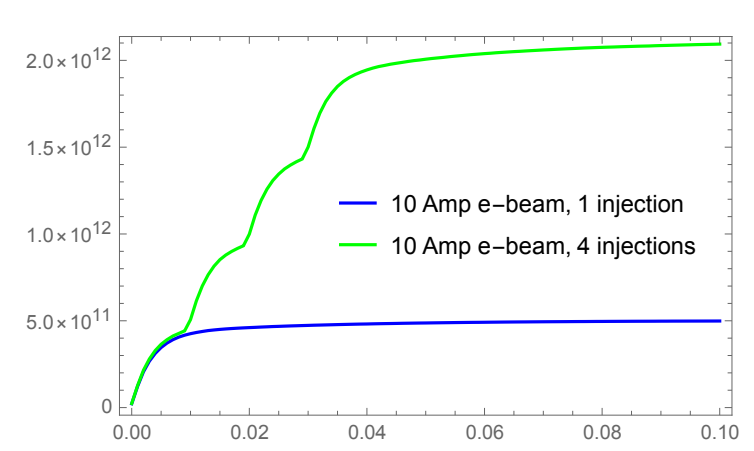

(a) Ionized ${ }^{3} \mathrm{He}$.

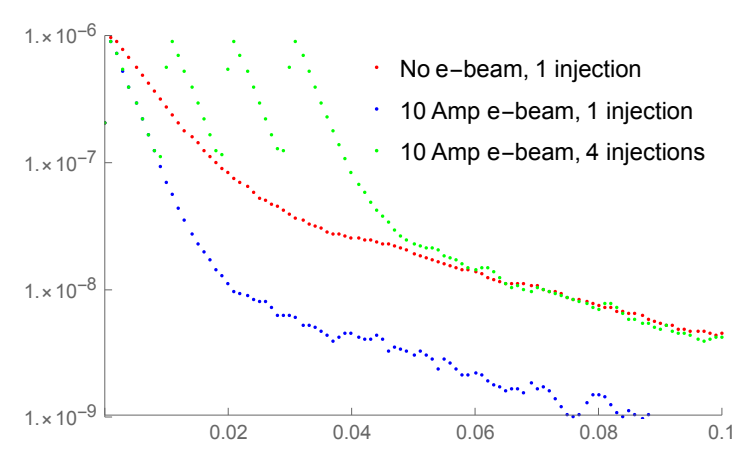

(b) Pressure (torr).

Figure 3: The ionized ${ }^{3} \mathrm{He}$ atoms and ionization cell pressure versus time (seconds) after injection of $8.3 \times 10^{11}{ }^{3} \mathrm{He}$ atoms per injection pulse. The number of ionized ${ }^{3} \mathrm{He}$ atoms trapped by the EBIS will be limited by the charge capacity of the e-beam.

In one simulated configuration, the gas ionization cell is a $1 \mathrm{~cm}$ diameter $30 \mathrm{~cm}$ long drift tube with $0.5 \mathrm{~cm}$ constrictions at the ends and differential pumping. The small diameter will limit the amount of gas that needs to be injected into the EBIS to neutralize the electron beam, and the constrictions will limit gas flow from the gas ionization cell to the EBIS high vacuum regions. Simulations with MolFlow (a Monte Carlo simulator for high vacuum) [19] show that the injected ${ }^{3} \mathrm{He}$ will diffuse into the ionizing region in $2 \mathrm{~ms}$, and the $10 \mathrm{Amp}$ electron beam will ionize ${ }^{3} \mathrm{He}$ to ${ }^{3} \mathrm{He}^{+}$with $\sim 60 \%$ efficiency in $<10 \mathrm{~ms}$. If more ${ }^{3} \mathrm{He}$ gas needs to be injected to neutralize the electron beam while keeping the pressure below $10^{-6}$ torr in the ionization cell, multiple gas injections can be used, see Figure 3a. In order to prevent build up of ${ }^{3} \mathrm{He}$ gas during consecutive pulses and enable fast switching of ion species, injected gas needs to be pumped out and the vacuum brought from $10^{-6}$ to $10^{-9}$ torr on a time scale of $200 \mathrm{~ms}$, see Figure $3 \mathrm{~b}$ for estimated pump down times of the ionization region.

After ${ }^{3} \mathrm{He}$ is ionized to ${ }^{3} \mathrm{He}^{+}$, the ${ }^{3} \mathrm{He}^{+}$ions quickly move downstream in less than $1 \mathrm{~ms}$ where conversion to ${ }^{3} \mathrm{He}^{++}$will take place along the $1.5 \mathrm{~m}$ length of the ion trap in the downstream EBIS magnet. Separation of the gas ionization and ion trap regions reduces the influence of charge exchange processes and improves the ${ }^{3} \mathrm{He}^{++} \beta \mathrm{He}^{+}$ratio. This separation is accomplished with potential barriers that limit the return of ${ }^{3} \mathrm{He}^{+}$and ${ }^{3} \mathrm{He}^{++}$ions to the gas ionization cell. The conversion of $5 \times 10^{113} \mathrm{He}^{+}$to ${ }^{3} \mathrm{He}^{++}$in the $10 \mathrm{Amp}\left(575 \mathrm{~A} / \mathrm{cm}^{2}\right)$ e-beam occurs with an exponential time constant of $1 \mathrm{~ms}$ (see Figure 4), so the initial ionization of ${ }^{3} \mathrm{He}$ gas will be the limiting rate of production of ${ }^{3} \mathrm{He}^{++}$. In practice, the rate of ionization and conversion of ${ }^{3} \mathrm{He}^{+}$to ${ }^{3} \mathrm{He}^{++}$can be adjusted by varying the e-beam parameters. It will be necessary to limit the amount of injected ${ }^{3} \mathrm{He}$ gas so that further ionization of ${ }^{3} \mathrm{He}$ after neutralization of the e-beam does not cause ${ }^{3} \mathrm{He}^{++}$ to be ejected from the ion trap downstream in the high vacuum region of EBIS. Time scales for the production of polarized ${ }^{3} \mathrm{He}^{++}$in EBIS are shown in Table 2. 


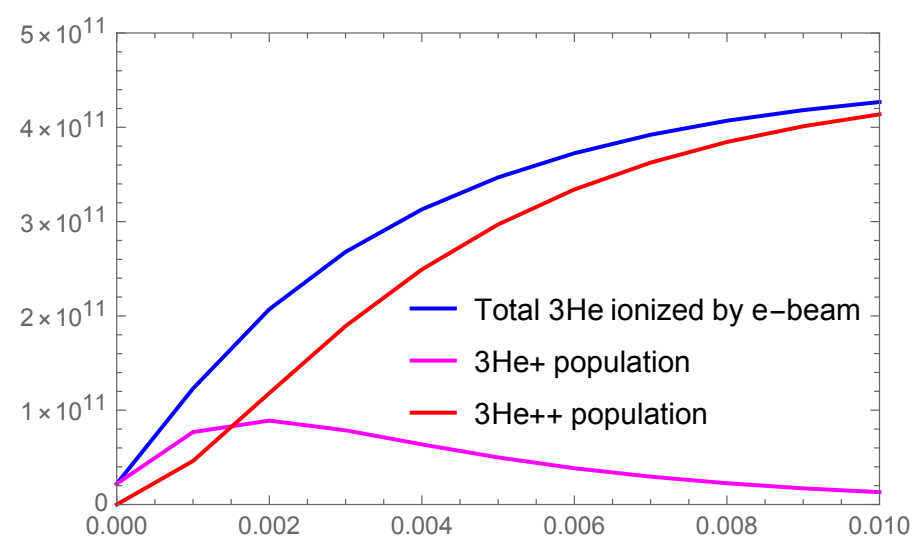

Figure 4: Ionization of ${ }^{3} \mathrm{He}$ gas and conversion of ${ }^{3} \mathrm{He}^{+}$to ${ }^{3} \mathrm{He}^{++}$in a $10 \mathrm{Amp}, 20 \mathrm{keV}$ e-beam (time in seconds). Charge exchange processes are ignored.

Table 2: EBIS polarized ${ }^{3} \mathrm{He}^{++}$production with $10 \mathrm{Amp}, 20 \mathrm{keV}$ e-beam

\begin{tabular}{lc}
\hline Step sequence & Time \\
\hline${ }^{3} \mathrm{He}$ gas injection & $0.5 \mathrm{~ms}$ \\
Diffusion into ionization cell & $2 \mathrm{~ms}$ \\
Injected gas pressure falls $50 \%$ & $3 \mathrm{~ms}$ \\
Ionization of ${ }^{3} \mathrm{He} \mathrm{to}^{3} \mathrm{He}^{+}$ & $\sim 10$ ms per gas injection \\
Time constant for ${ }^{3} \mathrm{He}^{+} \rightarrow{ }^{3} \mathrm{He}^{++}$conversion & $1 \mathrm{~ms}$ \\
Pump down to $10^{-9}$ torr & $100-150 \mathrm{~ms}$ \\
5 Hz EBIS pulse repetition rate & $200 \mathrm{~ms}$ \\
Switching time between species & 1 second \\
\hline
\end{tabular}

\section{Low-Energy ${ }^{3} \mathrm{He}^{++}$Beam Polarimetry}

The performance of the EBIS based ${ }^{3} \mathrm{He}^{++}$ion source will be tested by measuring the ${ }^{3} \mathrm{He}$ polarization at $6 \mathrm{MeV}$ after acceleration through the EBIS linac via scattering on an unpolarized ${ }^{4} \mathrm{He}$ gas target. A concept low-energy polarimeter design is shown in Figure 5. The analyzing power for elastic scattering of polarized ${ }^{3} \mathrm{He}$ on unpolarized ${ }^{4} \mathrm{He}$ is ideally $A_{N}=1$ at a beam energy of $\sim 5.3 \mathrm{MeV}$ and $\theta_{C M} \approx 91^{\circ}[20,21]$. For the $6 \mathrm{MeV}$ polarized ${ }^{3} \mathrm{He}^{++}$beam emitted from the EBIS linac, there is a local maximum at $A_{N}>0.9$ and $\theta_{C M} \approx 96^{\circ}$ where the polarization can be determined by measuring the spin correlated asymmetry from scattered ${ }^{3} \mathrm{He}$ and ${ }^{4} \mathrm{He}$. The ${ }^{3} \mathrm{He}^{++}$ polarization state will be set with a spin rotator in the High Energy Beam Transport (HEBT) line after the EBIS linac, which will align the ${ }^{3} \mathrm{He}^{++}$polarization to the transverse vertical direction at $6 \mathrm{MeV}$ beam energy.

One proposed layout for the spin direction alignment system is shown in Figure 6. The ${ }^{3} \mathrm{He}$ longitudinal polarization is first rotated to the transverse direction by the $21.5^{\circ}$ bending magnet (1). Then the solenoid spin-rotator rotates the spin to the vertical direction. The spin-rotator will be a pulsed solenoid with a reversible field to enable spin flip of EBIS pulses on a pulse-by-pulse 


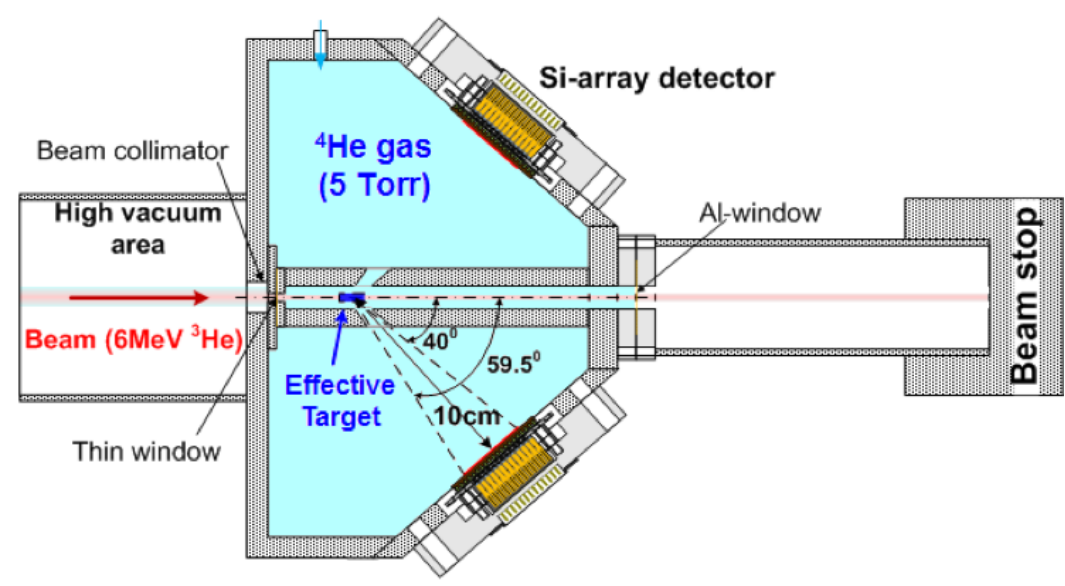

Figure 5: Concept for a low-energy ${ }^{3} \mathrm{He}$ polarimeter.

basis. The vertically polarized beam can be returned back to the straight HEBT line by the system of three dipole magnets $(2,3,4)$ after the spin rotator solenoid. Beam quality will be preserved with a number of focusing quadrupoles, steering magnets, and beam diagnostics. The low-energy ${ }^{3} \mathrm{He}$ polarimeter can be installed in the straight beam line section after the dipole magnet (3).

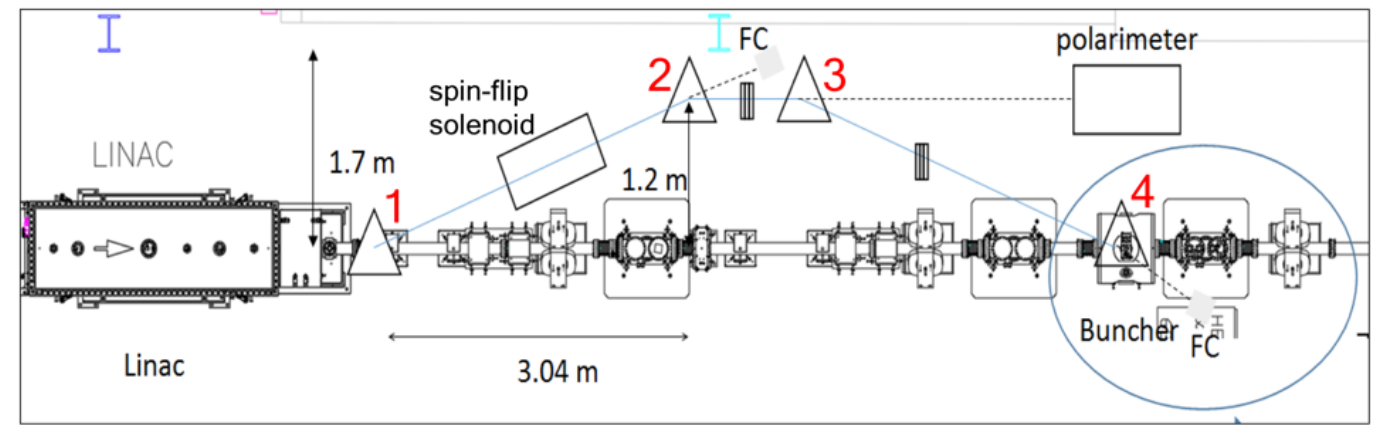

Figure 6: Concept for a ${ }^{3} \mathrm{He}$ spin rotator.

Inside the low-energy ${ }^{3} \mathrm{He}$ polarimeter, scattered ${ }^{3} \mathrm{He}$ and ${ }^{4} \mathrm{He}$ particles will be detected by silicon photodiode strip detectors over a range of center-of-mass scattering angles $\left(69^{\circ}<\theta_{C M}<100^{\circ}\right)$ and recoil particle energies (2.6-4.2 MeV for ${ }^{3} \mathrm{He}$ and 1.5-2.4 MeV for ${ }^{4} \mathrm{He}$ ). Simultaneous detection of scattered ${ }^{3} \mathrm{He}$ and ${ }^{4} \mathrm{He}$ provides a coincidence signal to reduce background. To produce an absolute polarization measurement, the polarimeter will be calibrated at $5.3 \mathrm{MeV}$ either by decelerating the ${ }^{3} \mathrm{He}^{++}$beam after the EBIS linac with a buncher or by detuning the EBIS linac to increase the energy spread. The ${ }^{4} \mathrm{He}$ gas target will be at 5 torr with an effective target length of $1 \mathrm{~cm}$. The statistical error for the ${ }^{3} \mathrm{He}$ polarization measurement is estimated at $(\delta P / P)_{\text {stat }} \approx 1.1 \% / \mathrm{min}$. In addition to testing the ${ }^{3} \mathrm{He}^{++}$ion source, this feasibility experiment will set a benchmark for ${ }^{3} \mathrm{He}$ beam polarimetry at higher energies farther down the accelerator chain, which is another area of necessary R\&D for the realization of an EIC. 


\section{Acknowledgments}

This research is supported by the Program for R\&D for Next Generation Nuclear Physics Accelerator Facilities of the DOE Office of Nuclear Physics under contract numbers DE-SC0008740 and DE-SC0012704.

\section{References}

[1] W. E. Burcham, O. Karban, S. Oh and W. B. Powell, A source of polarized 3He ions, Nuclear Instruments and Methods 116 (1974) 1.

[2] D. Findley, S. Baker, E. Carter and N. Stockwell, A polarized 3He+ ion source, Nuclear Instruments and Methods 71 (1969) 125.

[3] R. Slobodrian, C. Rioux, J. Giroux and R. Roy, A polarized 3He ion source for electrostatic accelerators, Nuclear Instruments and Methods in Physics Research Section A: Accelerators, Spectrometers, Detectors and Associated Equipment 244 (1986) 127.

[4] Report of the electron ion collider advisory committee, tech. rep., 2009.

[5] Report of the Community Review of EIC Accelerator R\&D for the Office of Nuclear Physics, tech. rep., DOE Office of Nuclear Physics, 2017.

[6] C. S. Epstein, Development of a polarized 3 He ion source for RHIC, AIP Conference Proceedings 1441 (2012) 643.

[7] C. S. Epstein, Development of a Polarized Helium-3 Ion Source for RHIC using the Electron Beam Ion Source, B. S. Thesis, Massachusetts Institute Of Technology, 2013.

[8] J. D. Maxwell, C. S. Epstein and R. G. Milner, Diffusive Transfer of Polarized 3He Gas through Depolarizing Magnetic Gradients, 1412.6167.

[9] J. D. Maxwell, Development of a Polarized 3He Beam Source for RHIC with EBIS, in PSTP2015, 2015.

[10] J. Maxwell, C. Epstein, R. Milner, J. Alessi, E. Beebe, A. Pikin et al., Development of a Polarized Helium-3 Source for RHIC and eRHIC, International Journal of Modern Physics: Conference Series 40 (2016) 1660102.

[11] J. Alessi, Electron Beam Ion Source Pre-Injector Project, Tech. Rep. BNL-73700-2005-IR, Brookhaven National Laboratory, 2005.

[12] A. Zelenski and J. Alessi, Beam Dynamics Newsletter, .

[13] A. Zelenski, J. Alessi, A. Kponou and D. Raparia, High-intensity polarized H- (PROTON), deuteron and $3 H E++$ ion source development at BNL, EPAC 2008 - Contributions to the Proceedings (2008) 1010.

[14] A. Pikin, A. Zelenski, A. Kponou, J. Alessi, E. Beebe, K. Prelec et al., Ionization of polarized 3He+ ions in EBIS trap with slanted electrostatic mirror, AIP Conference Proceedings 980 (2008) 248.

[15] T. R. Gentile, P. J. Nacher, B. Saam and T. G. Walker, Optically polarized He3, Reviews of Modern Physics 89 (2017) [1612.04178].

[16] A. Nikiel-Osuchowska, G. Collier, B. Głowacz, T. Pałasz, Z. Olejniczak, W. P. Wȩglarz et al., Metastability exchange optical pumping of 3 He gas up to hundreds of millibars at 4.7 tesla, European Physical Journal D 67 (2013) . 
[17] I. Alekseev, C. Allgower, M. Bai and E. Al., Configuration Manual Polarized Proton Collider at RHIC, tech. rep., Brookhaven National Laboratory, January, 2006.

[18] E. N. Beebe, J. G. Alessi, D. Graham, A. Kponou, A. Pikin, K. Prelec et al., Test EBIS operation and component development for the RHIC EBIS*, Journal of Physics: Conference Series 2 (2004) 164.

[19] R. Kersevan and J.-L. Pons, Introduction to MOLFLOW+: New graphical processing unit-based Monte Carlo code for simulating molecular flows and for calculating angular coefficients in the compute unified device architecture environment, Journal of Vacuum Science \& Technology A: Vacuum, Surfaces, and Films 27 (2009) 1017.

[20] D. Hardy, R. Spiger, S. Baker, Y. Chen and T. Tombrello, Polarization in 3 He +4 He elastic scattering, Physics Letters B 31 (1970) 355.

[21] D. M. Hardy, R. J. Spiger, S. D. Baker, Y. S. Chen and T. A. Tomberllo, Scattering of 3He and $4 H e$ from polarized 3He between 7 and 18 MeV, Nuclear Physics, Section A 195 (1972) 250. 